\title{
Modulational Instability in Bose-Einstein Condensates under Feshbach Resonance Management
}

\author{
Z. Rapti ${ }^{1}$, G. Theocharis ${ }^{2}$, P.G. Kevrekidis ${ }^{1}$, D.J. Frantzeskakis ${ }^{2, *}$, and B.A. Malomed ${ }^{3}$ \\ ${ }^{1}$ Department of Mathematics and Statistics, University of Massachusetts, Amherst MA 01003-4515, USA \\ ${ }^{2}$ Department of Physics, University of Athens, Panepistimiopolis, Zografos, Athens 15784, Greece \\ ${ }^{3}$ Department of Interdisciplinary Studies, Faculty of Engineering, Tel Aviv University, Tel Aviv 69978, \\ Israel
}

\begin{abstract}
We investigate the modulational instability of nonlinear Schrödinger equations with periodic variation of their coefficients. In particular, we focus on the case of the recently proposed, experimentally realizable protocol of Feshbach Resonance Management for Bose-Einstein condensates. We derive the corresponding linear stability equation analytically and we show that it can be reduced to a Kronig-Penney model, which allows the determination of the windows of instability. The results are tested numerically in the absence, as well as in the presence of the magnetic trapping potential.
\end{abstract}

PACS numbers: 03.75.-b, 03.75.Kk, 03.75.Lm

*Email address: dfrantz@cc.uoa.gr 


\section{Introduction}

The experimental realization and intense theoretical studies of Bose-Einstein condensates (BECs) [1] have led to an explosion of interest in the field of atomic matter waves and nonlinear excitations in them. In particular, one-dimensional (1D) dark [2] and bright [3] solitons have been created in experiments, in BECs with repulsive and attractive interactions, which correspond to the positive and negative atom-atom scattering length (SL), respectively. Additionally, multi-dimensional solitons [4, Faraday waves [5], ring dark solitons and vortex necklaces [6], have been theoretically predicted. The BEC solitons are of fundamental interest, not only conceptually, but also practically: one can envision robust solitary structures being coherently manipulated in matter-wave chips, similar to how light is controlled in the existing optical ones [7].

To generate BEC solitons in one spatial dimension (in the presence of the magnetic trap [8, 9], or in the quasi-discrete setting created by an optical-lattice potential [10]), as well to avoid their collapse in two dimensions [11, an experimentally realizable protocol has been recently proposed, in the form of the so-called Feshbach Resonance Management (FRM). It is based on adjusting the effective SL (including a possibility to change its sign) by a resonantly tuned ac magnetic field through the Feshbach resonance [12.

The FRM scheme can be modelled (in the mean-field approximation) in the framework of the GrossPitaevskii (GP) equation [1, with the coefficient in front of the nonlinear term being a periodic function of time. In Ref. [8], the periodic function has been taken to be a piece-wise constant one, periodically jumping between positive and negative values. The same model may also be realized in terms of nonlinear optics, where it applies to a medium composed of alternating layers with opposite signs of the Kerr nonlinearity 13. FRM resembles the dispersion-management (DM) scheme, well-known in fiber optics (see, e.g., 14] and [15] for review), which assumes that nonlinear fibers with opposite signs of the group-velocity dispersion periodically alternate, to form a system supporting robust breathing solitons. Similarly to this, very robust and stable matter-wave breathing solitons can be generated by means of the FRM in BECs [], which lead to structures (such as, e.g., the dark solitons) even more robust than their optical counterparts [16].

The purpose of this paper is to study how solitary waves can arise in the FRM setting and, in particular, to investigate the modulational instability (MI) in the corresponding nonlinear Schrödinger (NLS) equations with periodically varying coefficients. Such a study is relevant, as MI is one a fundamental mechanism that leads to the formation of localized solitary-wave structures in a variety of settings, ranging from fluid dynamics (where it is usually referred to as the Benjamin-Feir instability) [17] to nonlinear optics [18] and plasma physics [19, and, most recently, to BECs in the presence of an optical lattice [20] or magnetictrap potentials [21]. In the present work, the study of the MI leads to a general linear stability equation which includes, as special cases, equations previously examined in the context of DM. We then proceed to analyze MI conditions in the case of the piecewise-constant FRM scheme [8], and find that the resulting stability equation resembles the well-known Kronig-Penney model of solid-state physics [22]. Thus, we derive analytical criteria for the MI.

The paper is organized as follows. The derivation of the stability equation and its analytical treatment are presented in Section 2. In Section 3, we turn to numerical experiments both in the absence of the 
magnetic trap (the case for which we have analytical results) and in the presence of it, as the magnetic trap is a necessary ingredient of the experiment. In Section 4 we summarize our findings and present our conclusions.

\section{Stability analysis}

We consider a general GP/NLS equation, with time-dependent coefficients in front of the dispersive and the nonlinear terms, $D(t)$ and $a(t)$, respectively. The equation also includes a term corresponding to the external magnetic (parabolic) potential trap:

$$
i u_{t}=-D(t) u_{x x}+a(t)|u|^{2} u+(1 / 2) \Omega^{2} x^{2} u
$$

In the case of BECs $(D=$ const $\equiv 1 / 2), u(x, t)$ is the macroscopic wave function, $x$ and $t$ are measured in the harmonic-oscillator units, and $\Omega$ is the strength of the magnetic trap [1]. If the FRM is applied [8], the nonlinear coefficient $a(t)$ periodically alternates between $a_{1}$ (for $0<t \leq \tau$ ) and $-a_{2}$ (for $\tau<t \leq T$ ), where it is assumed that $a_{1}, a_{2}>0$. As Eq. (11) is spatially inhomogeneous due to the presence of the parabolic trap, analytical investigation of the linear stability of the uniform states is only possible for $\Omega=0$. Hence, we will examine this case, which will be complemented by numerical results for both $\Omega=0$ and $\Omega \neq 0$.

The plane-wave solution to Eq. (1) is

$$
u_{0}=A_{0} \exp \left[i\left(-q^{2} \int_{0}^{t} D(s) d s-A_{0}^{2} \int_{0}^{t} a(s) d s+q x\right)\right] .
$$

Notice that the solution's amplitude can be rescaled to $A_{0} \equiv 1$. Then, a solution including an infinitesimal perturbation is sought as

$$
u=u_{0}[1+\epsilon w(t) \cos (k x)]
$$

where $\epsilon$ and $k$ are the amplitude and wavenumber of the perturbation, which leads to the following linearized equations for $w \equiv w_{r}+i w_{i}$ :

$$
\dot{w}_{r}=k^{2} D(t) w_{i}, \dot{w}_{i}=-\left[k^{2} D(t)+2 a(t)\right] w_{r}
$$

which can be transformed into a single equation,

$$
\ddot{w}_{r}=\dot{D} D^{-1} \dot{w}_{r}-k^{2} D(t)\left[k^{2} D(t)+2 a(t)\right] w_{r}
$$

the overdot standing for $d / d t$.

There are several special cases of this equation that were previously studied. In the case of DM (i.e., for $D=D(t)$ and $a(t) \equiv$ const), the MI analysis was performed in [23]. On the other hand, in the FRM context, for $D \equiv 1 / 2$ and time-periodic $a(t)$, Eq. (2] is a Hill equation that was considered in [8], while the more specialized case of $a(t)=1+2 \alpha \cos (\omega t)$ leads to the Mathieu equation that was dealt with, in this context, in 5 .

Motivated by the FRM scheme proposed in [8], we will now explore the special case of a piecewiseconstant time-dependent SL (alternating between $a_{1}$ and $-a_{2}$ as discussed above). In this case, we define 
$s_{1}^{2}=k^{2}\left(k^{2} / 4+a_{1}\right)$ and $s_{2}^{2}=k^{2}\left(a_{2}-k^{2} / 4\right)$, which assumes $k^{2}<4 a_{2}$. Searching for a solution in accordance with Bloch's theorem [22], we find, within one period,

$$
\begin{aligned}
& w_{r}=A e^{i\left(-\omega+s_{1}\right) t}+B e^{i\left(-\omega-s_{1}\right) t}, \quad \text { for } 0<t \leq \tau, \\
& w_{r}=C e^{\left(-i \omega+s_{2}\right) t}+D e^{\left(-i \omega-s_{2}\right) t}, \quad \text { for } \tau \leq t \leq T .
\end{aligned}
$$

Then one should request the continuity of the solution and its derivative at $t=0$ and $t=\tau$, obtaining 4 conditions on $A, B, C, D$, namely,

$$
\begin{aligned}
e^{i s_{1} \tau} A+e^{-i s_{1} \tau} B-e^{s_{2} \tau} C-e^{-s_{2} \tau} D & =0, \\
i\left(-\omega+s_{1}\right) e^{i s_{1} \tau} A+i\left(-\omega-s_{1}\right) e^{-i s_{1} \tau} B-\left(-i \omega+s_{2}\right) e^{s_{2} \tau} C-\left(-i \omega-s_{2}\right) e^{-s_{2} \tau} D & =0, \\
A+B-e^{\left(-i \omega+s_{2}\right) T} C-e^{\left(-i \omega-s_{2}\right) T} D & =0, \\
i\left(-\omega+s_{1}\right) A+i\left(-\omega-s_{1}\right) B-\left(-i \omega+s_{2}\right) e^{\left(-i \omega+s_{2}\right) T} C-\left(-i \omega-s_{2}\right) e^{\left(-i \omega-s_{2}\right) T} D & =0 .
\end{aligned}
$$

The resulting system of homogeneous linear equations for $(A, B, C, D)$ has non-trivial solutions only if its determinant vanishes. This condition leads to the following equation for the eigenfrequency (Floquet multiplier) $\omega$ :

$$
\cos (\omega T)=-\frac{s_{1}^{2}-s_{2}^{2}}{2 s_{1} s_{2}} \sin \left(s_{1} \tau\right) \sinh \left[s_{2}(T-\tau)\right]+\cos \left(s_{1} \tau\right) \cosh \left[s_{2}(T-\tau)\right] \equiv F(k) .
$$

If the above condition $k^{2}<4 a_{2}$ does not hold, we redefine $\tilde{s}_{2}=\sqrt{k^{2}\left(k^{2} / 4-a_{2}\right)}$, and obtain, instead of Eq. (5),

$$
\cos (\omega T)=-\frac{s_{1}^{2}+\tilde{s}_{2}^{2}}{2 s_{1} \tilde{s}_{2}} \sin \left(s_{1} \tau\right) \sin \left[\tilde{s}_{2}(T-\tau)\right]+\cos \left(s_{1} \tau\right) \cos \left[\tilde{s}_{2}(T-\tau)\right] \equiv \widetilde{F}(k) .
$$

By examining the function $|F(k)|$ or $|\widetilde{F}(k)|$, defined in Eqs. (5) and (6), and comparing it to 1, we can find whether there is a real eigenfrequency for a given perturbation wavenumber $k$, or it belongs to a "forbidden zone", which implies the MI.

A typical example of results produced by this analysis is shown in Fig. 1 for $a_{1}=a_{2}=0.3$ and $\tau=T / 4=1$. The first several instability windows, which are determined by the shape of the curve $F(k)$ for this case are given in Table I. Notice that in the table, only positive values of unstable wavenumbers are given, the negative ones obeying the $k \rightarrow-k$ symmetry. In general, we have found that the number and widths of the instability windows increase as long as the mean value $\bar{a} \equiv\left[a_{1} \tau-a_{2}(T-\tau)\right] / T$ of the SL gets large and negative, i.e., when the BEC is "very attractive" on the average.

\section{Numerical Results}

We now examine the validity of the above theoretical predictions through full numerical simulations of Eq. (11). This also allows us to investigate the role of the magnetic trap that was not taken into regard in the above analysis.

As a typical example (similar results have been obtained in other cases), we consider modulationally stable and unstable cases for the example presented above (i.e., $a_{1}=a_{2}=0.3$ and $\tau=T / 4=1$.). In particular, we examine the case of $k=0.5$ which, according to Fig. 1 and Table I, should be unstable, and the case 
of $k=1$, that is expected to be stable. It can be clearly observed that the corresponding configurations evolve in accordance with the theoretical prediction, indeed leading to the MI and, as a result, generation of a lattice of solitary waves, for $k=0.5$. Instead, the modulational perturbations remain small for $k=1$. The agreement between the analytical predictions and direct simulations has been found to be generic.

We now proceed to the case when a weak magnetic trap is included, taking (for example) $\Omega^{2}=0.00025$. Then we observe that both the configurations which were linearly stable and unstable in the absence of the trap eventually develop the MI, which is in consonance also with the findings of [21]. Here, we used the initial condition $u=u_{\mathrm{TF}}[1+0.02 \cos (k x)]$, where $u_{\mathrm{TF}}=\sqrt{\max (0, \mu-V(x))}$ is the wave function in the ThomasFermi approximation [1]. The reason why the MI develops in both cases is that the magnetic trap mixes stable and unstable wavenumbers in the Fourier space. As a result, even though the initial configuration contained only stable wavenumbers, unstable ones are eventually generated, giving rise the MI. However, as can be observed in Fig. 3 the MI occurs faster in the case that would be modulationally unstable in the absence of the trap, as the MI growth rate is larger in this case than in the case when the uniform state was stable in the absence of the trap.

We also examined what happens with the increase of the strength of the magnetic trap, which makes it "tighter". In this case, the trapping potential is much more efficient in mixing the wavenumbers, therefore there remains little difference between the evolution of configurations that were modulationally unstable and

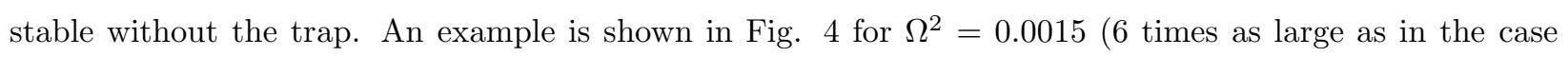
shown in Fig. 3). It can be seen that the development of the MI for $k=0.5$ and $k=1$ is practically identical.

\section{Conclusions}

In this paper, we have studied the modulational stability (MI) in the GP/NLS equations with periodically varying coefficients. We highlighted how the results may be applied to problems stemming from both nonlinear optics and Bose-Einstein condensation. Findings reported in earlier works in the subject have been generalized. The equation obtained from the analysis, Eq. (2), is of interest in its own right. Motivated by the recently introduced concept of a periodically modulated (via a Feshbach resonance) scattering length as a means of generating robust breathing matter-wave solitons [8], we have focused attention on the case of alternating positive and negative scattering length.

For the case of the FRM, we have demonstrated that the above-mentioned equation governing the MI development is an equation of the Hill type. More specifically, it is an analog of the Kronig-Penney model known in solid state physics. Then, developing analysis similar to the eigenvalue calculation in the KronigPenney model, we have obtained analytical conditions for the MI of such FRM schemes. The analytical criteria have been shown to be in agreement with full numerical simulations. Although the analysis was carried out in the absence of the magnetic trap, the role of the trap was investigated numerically. For weak trap potentials, it was found that the MI will always set in (due to the mixing of wavenumbers imposed by the spatial potential); however, its growth rate bears "memory" of the presence/absence of the MI in the corresponding situation without the trap. On the other hand, if the trap is strong, the memory is effectively wiped out. 


\section{Acknowledgements}

This work was partially supported by a UMass FRG, NSF-DMS-0204585 and the Eppley Foundation for Research (PGK), as well as by the Special Research Account of the University of Athens under grant No. $70 / 4 / 5844$ (GT, DJF).

\section{References}

[1] F. Dalfovo et al., Rev. Mod. Phys. 71, 463 (1999).

[2] S. Burger et al., Phys. Rev. Lett. 83, 5198(1999); J. Denschlag et al., Science 287, 97 (2000); B. P. Anderson et al., Phys. Rev. Lett. 86, 2926 (2001).

[3] K.E. Strecker et al., Nature 417, 150 (2002); L. Khaykovich et al., Science 296, 1290 (2002).

[4] B.B. Baizakov, V.V. Konotop, and M. Salerno, J. Phys. B 35, 5105 (2002); E.A. Ostrovskaya and Y.S. Kivshar, Phys. Rev. Lett. 90, 160407 (2003).

[5] K. Staliunas, S. Longhi and G. J. de Valcárcel, Phys. Rev. Lett. 89, 210406 (2002).

[6] G. Theocharis et al., Phys. Rev. Lett. 90, 120403 (2003).

[7] R. Folman and J. Schmiedmayer, Nature 413, 466 (2001); R. Folman et al., Adv. Atom. Mol. Opt. Phys. 48, 263 (2002).

[8] P.G. Kevrekidis et al., Phys. Rev. Lett. 90, 230401 (2003).

[9] F.Kh. Abdullaev et al., Phys. Rev. Lett. 90, 230402 (2003).

[10] F.Kh. Abdullaev et al., cond-mat/0306281

[11] F. Kh. Abdullaev et al., Phys. Rev. A 67, 013605 (2003); H. Saito and M. Ueda, Phys. Rev. Lett. 90, 040403 (2003).

[12] S. Inouye et al., Nature 392, 151 (1998); E.A. Donley et al., Nature 412, 295 (2001).

[13] I. Towers and B.A. Malomed, J. Opt. Soc. Am. B 19, 537 (2002).

[14] B.A. Malomed, Progress in Optics 43, 71 (2002).

[15] S.K. Turitsyn et al, C.R. Physique 4, 145 (2003).

[16] C. Paré and P.-A. Bélanger, Opt. Commun. 168, 103 (1999).

[17] T.B. Benjamin and J.E. Feir, J. Fluid. Mech. 27, 417 (1967).

[18] L.A. Ostrovskii, Sov. Phys. JETP 24, 797 (1969).

[19] T. Taniuti and H. Washimi, Phys. Rev. Lett. 21, 209 (1968); A. Hasegawa, Phys. Rev. Lett. 24, 1165 (1970). 
[20] B. Wu and Q. Niu, Phys. Rev. A 64, 061603(R) (2001); V. V. Konotop, and M. Salerno, Phys. Rev. A 65, 021602 (2002); A. Smerzi et al Phys. Rev. Lett., 89, 170402 (2002).

[21] G. Theocharis et al., Phys. Rev. A 67, 063610 (2003); L.D. Carr and J. Brand, cond-mat/0303257

[22] C. Kittel, Introduction to Solid State Physics (John Wiley and Sons: New York, 1986).

[23] N.J. Smith and N.J. Doran, Opt. Lett. 21, 570 (1996); F.Kh. Abdullaev et al., Phys. Lett. A 220, 213 (1996). 


\begin{tabular}{|l|l|}
\hline Window & $\mathrm{k}$ \\
\hline 1 & {$[0,0.7489)$} \\
\hline 2 & $(1.2502,1.4957)$ \\
\hline 3 & $(1.8283,1.9115)$ \\
\hline 4 & $(2.2396,2.2562)$ \\
\hline 5 & $(2.5638,2.5784)$ \\
\hline
\end{tabular}

Table I: The first instability bands of the perturbation wavenumber $k$ for the case $a_{1}=a_{2}=0.3$ and $\tau=T / 4=1$.

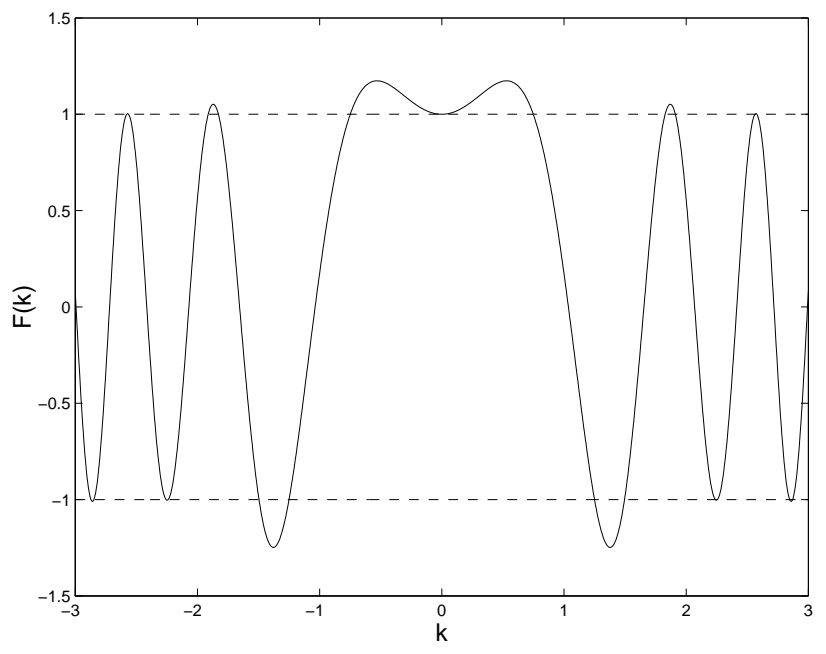

Figure 1: The plot of the function $F(k)$ defined by Eqs. (5) and (6) for $a_{1}=a_{2}=0.3$ and $\tau=T / 4=1$. When the function satisfies $|F(k)| \leq 1$, the perturbation of wavenumber $k$ is modulationally stable, while for $|F(k)|>1$, it is modulationally unstable. 
(a)
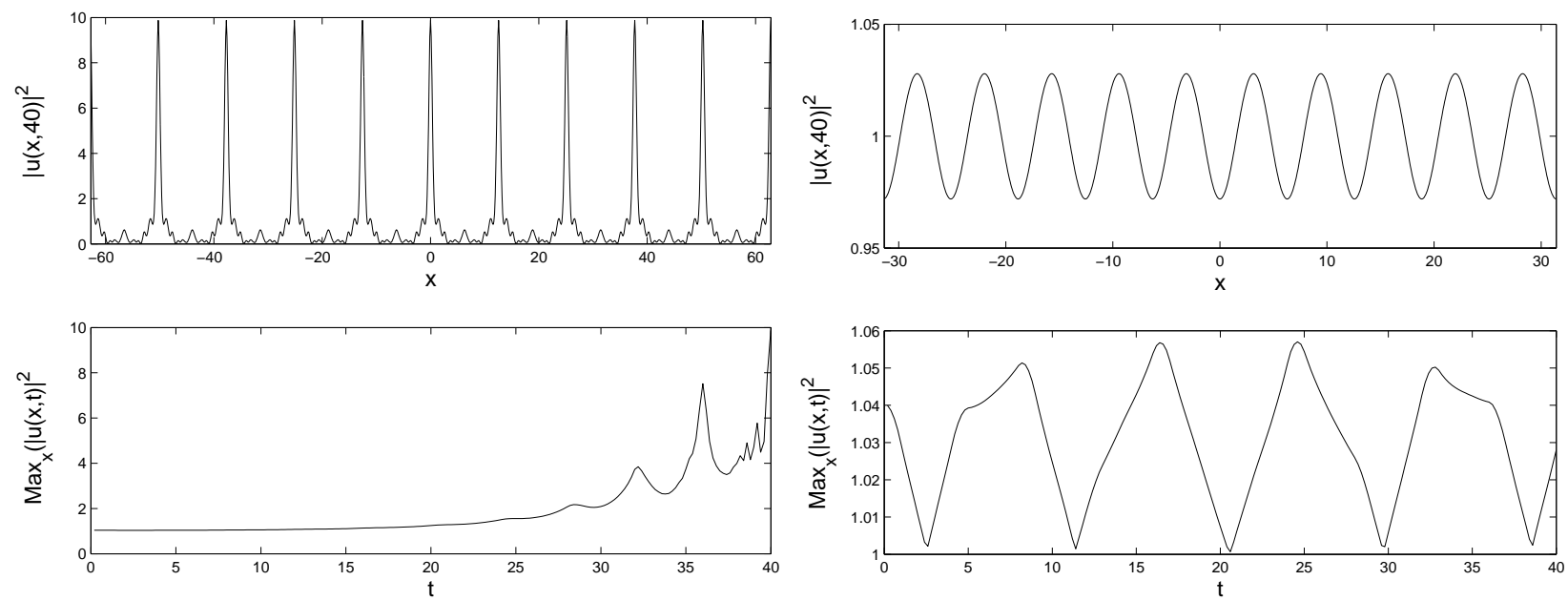

Figure 2: For the case $a_{1}=a_{2}=0.3$ and $\tau=T / 4=1, \Omega=0$, the evolution of modulationally unstable ( $k=0.5$, left panel) and stable $(k=1$, right panel) perturbations of the form $0.02 \cos (k x)$ added to $u=1$, is examined. The top and bottom panels show, respectively, the configuration at $t=40$ and the time evolution of the amplitude of the spatial profile.

(a)
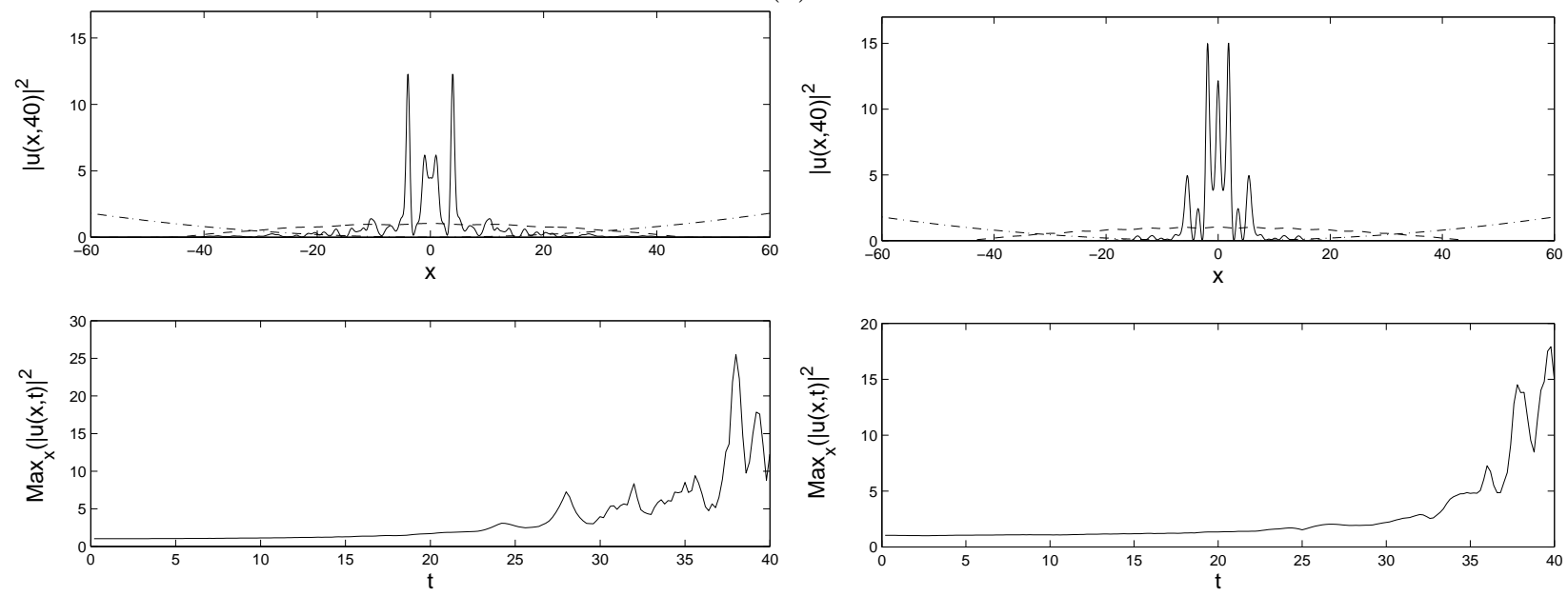

Figure 3: The same as in the previous figure, but now with $\Omega^{2}=0.00025$, the initial configuration being multiplied by the Thomas-Fermi wave function $\sqrt{\max (0, \mu-V(x))}$ with $\mu=1$. Notice that the modulational instability develops both for $k=0.5$ (left) and for $k=1$ (right). 
(a)
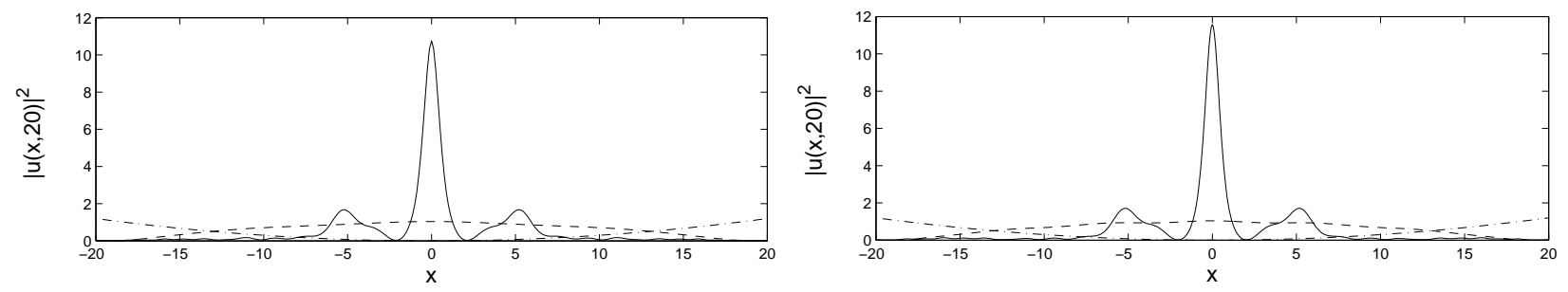

(b)


Figure 4: The same as in the previous figure, but with $\Omega^{2}=0.0015$. The only remaining visible difference between the cases of $k=0.5$ (left) and $k=1$ (right) is a slightly larger growth rate in the former case. 